\title{
GLOBULAR CLUSTERS IN NGC 1399 AND BACKGROUND GALAXIES ${ }^{1}$
}

\author{
Rainer Madejsky and Ralf Bender \\ Landessternwarte Königstuhl \\ D-6900 Heidelberg, FRG
}

\section{OBSERVATIONS AND RESULTS}

NGC 1399, the central galaxy of the Fornax cluster, is known to have an overabundance of globular clusters (Hanes and Harris, 1986). In order to determine the luminosity function of these globular clusters, two fields near NGC 1399 were observed in the B, V, and R band. One field was chosen near the center of NGC 1399 and the second field 20' east of the galaxy center. The second field is supposed to contain no globular clusters and is used for comparison. Both fields were reduced with DAOPHOT (Stetson, 1987) to determine the numbermagnitude relation for all detected objects (see Figure 1). The differential number counts give $\log N(m)=0.84 m_{\mathrm{B}}$ for the comparison field while the differential number counts for the central field (background galaxies and globular clusters) cannot be described by this simple law. Figure 2 shows the number-magnitude relation of the excess objects in the central field (difference between all counts in the central and in the comparison field). The differential number counts $N(m)$ of the excess objects can be represented by the superposition of three populations, each following approximately a Gaussian distribution. The population with the

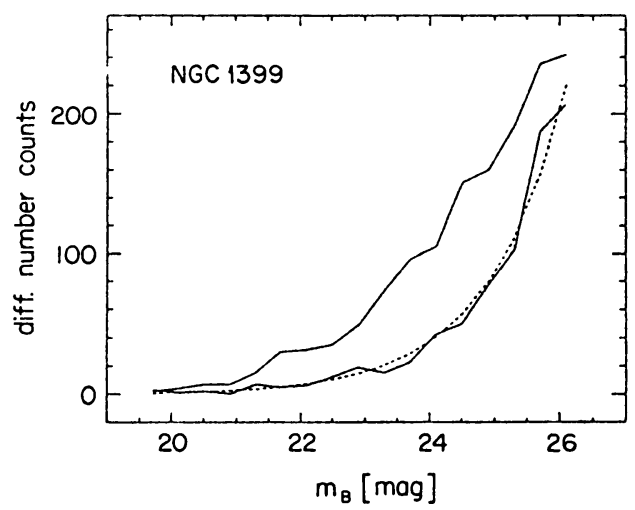

Figure 1. The differential number counts of the central field (upper line) and the comparison field (lower line) are displayed for the B band until $0.5 \mathrm{mag}$ short of the limiting magnitude. The differential number counts of the comparison field can be described by a simple exponential law; this function is displayed as a dotted line. The differential number counts of the central field cannot be approximated by a simple exponential function.

1 Based on observations collected at the European Southem Observatory, La Silla, Chile. 


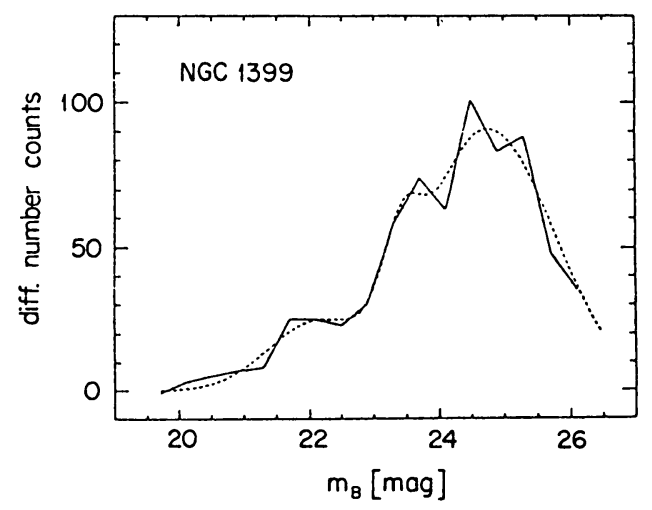

Figure 2. The difference among all detected objects between both fields $\left(N_{\text {cent }}(m)-N_{\text {comp }}(m)\right)$ is shown for the B band. The excess objects in the central field can be represented by the superposition of three populations. This three-component Gaussian model is indicated by the dotted line. The most abundant population $(N>250)$ with peak luminosity $m_{\mathrm{B}}=24.8 \mathrm{mag}$ is identified with globular clusters belonging to NGC 1399.

brightest peak luminosity $\left(m_{\mathrm{B}}=22.1 \mathrm{mag}\right)$ is due to a galaxy cluster with an estimated redshift $z=0.3-0.4$.

The second population has very similar color indices as the cluster at $z=0.3-0.4$ but the peak luminosity is $1.3 \mathrm{mag}$ fainter. This could be another and more distant cluster of galaxies, which presumably is very rich. Further analysis of color indices and the angular-diameterredshift relation is necessary in order to confirm this hypothesis. More than 250 objects belong to the third population with peak luminosity $m_{\mathrm{B}}=24.8 \mathrm{mag}, m_{\mathrm{V}}=24.0 \mathrm{mag}$, and $m_{\mathrm{R}}=23.4$ mag. We identify this population with globular clusters associated with NGC 1399 . The color indices are consistent with color indices of galactic globular clusters. The presence of probably two clusters of galaxies at different redshifts can contaminate the globular cluster population, but the number of detected globular clusters is sufficient to establish the peak luminosity with relatively high accuracy $\left(m_{B}=24.8 \mathrm{mag} \pm 0.2 \mathrm{mag}\right)$. Assuming a universal luminosity function for globular clusters with $M_{\mathrm{V} \text {,peak }}=-7.3 \mathrm{mag}$, we determine for NGC 1399 a distance modulus $(m-M)=31.3$. Further assuming NGC 1399 to be the dynamical center of the Fornax cluster $\left(v_{\text {rad }}=1300 \mathrm{~km} \mathrm{~s}^{-1}\right)$ and taking into account the peculiar motion of the Local Group (Yahil, Tammann, and Sandage, 1977; Sandage and Tammann, 1988) we suggest $H_{0}=$ $70 \mathrm{~km} \mathrm{~s}^{-1} \mathrm{Mpc}^{-1}$.

\section{REFERENCES}

Hanes, D. A. and Harris, W. E. 1986, Ap. J., 309, 564.

Sandage, A. and Tammann, G. A. 1988, Ap. J., 328, 1.

Stetson, P. B. 1987, Dominion Astrophysical Observatory, Victoria, British Columbia

Tyson, J. A. 1988, Astron. J., 96, 1.

Yahil, A., Tammann, G. A., and Sandage, A. 1977, Ap. J., 217, 903. 